Revue d'histoire de l'Amérique française

ZAS REVUE D.HISTOIRE DE L'AMÉRIQUE FRANÇAISE

\title{
Tutelle aux mineurs Le Moyne de Longueuil, 1755
}

\section{Jean-Jacques Lefebvre}

Volume 8, numéro 2, septembre 1954

URI : https://id.erudit.org/iderudit/301654ar

DOI : https://doi.org/10.7202/301654ar

Aller au sommaire du numéro

Éditeur(s)

Institut d'histoire de l'Amérique française

ISSN

0035-2357 (imprimé)

1492-1383 (numérique)

Découvrir la revue

Citer ce document

Lefebvre, J.-J. (1954). Tutelle aux mineurs Le Moyne de Longueuil, 1755. Revue d'histoire de l'Amérique française, 8(2), 258-260.

https://doi.org/10.7202/301654ar d'utilisation que vous pouvez consulter en ligne.

https://apropos.erudit.org/fr/usagers/politique-dutilisation/ 


\section{DOCUMENTS INÉDITS}

Nos ancêtres, riches ou pauvres? - Un exemple de 1755. - Une pièce contresignée du Gouverneur le Marquis de Duquesne. - Le beau style juridique du temps.

\section{TUTELLE AUX MINEURS LE MOYNE DE LONGUEUIL, 1755}

Lan mil Sept Cens Cinquante Cinq Le Deux may deux heures de relevée pard' nous jacques Guiton monrepos \& En la Chambre d'audiance est Comparu $\mathrm{m}^{\mathrm{re}}$ charles Lemoine Baron de Longueuil Capitaine djnfanterie fils aisné Et $p p^{\prime a l}$ heritier de feu $m^{\text {re }}$ Charles Lemoine Baron de longueuil chevalier de lordre militaire de St louis Gouverneur de cette ville et gouverneur de montréal et de feue dame Charlote Legoues degrais son épouse Lequel nous a dis qu'En Execution de notre ordonnance du jour dhyer Et avis au bas de la requete par luy avons presentée aux fins d'elire un tuteur a Ses frere Et Sœurs mineurs a leffet de proceder au partage des biens Et effets dependants desd. Successions il a pour' ce fait assembler en présence de haut et puissant Seigneur Monseigneur Le marquis duquesne chevalier de lordre Royal Et militaire de $\mathbf{S}^{\mathrm{t}}$ Louis Capitaine des vaisseaux du Roy Gouverneur Et lieutenant general pour le Roy En toute La nouvelle france $\mathrm{m}^{\text {re }}$ Gaspard adhemar de Lantagnac Ecuyer Chevalier dud. ordre militaire de St Louis Lieutenant de Roy de Cette ville ami desd. mineurs, pierre payen Ecuyer Sieur de noyan Chevalier dud. ordre militaire de $\mathrm{S}^{\mathrm{t}}$ Louis major de Cette ville Et gouverneur cousin desd. mineurs, jacques René Gaultier Ecuyer Seigneur de varenne Cousin desd. mineurs louis de Beaujeu Ecuyer $\mathrm{S}^{\mathrm{r}}$ de villemonde capitaine djnfanterie Cousin germain desd. mineurs joseph hector Devillerey Ecuyer $\mathbf{S}^{r}$ dartigny Enseigne d'jnfanterie Cousin desd. mineurs, jean le Ber Ec ayer $\mathrm{S}^{\mathbf{r}}$ de Senneville Enseigne djnfanterie cousin desd. mineurs $\mathrm{Sr}$ antoine Salvaye de tremont capitaine des portes de Cette ville Cousin desd. mineurs Et pierre de marganne Ecuyer Seigneur de lavaltrie chevalier de lordre militaire de $\mathrm{S}^{t}$ Louis capitaine djnfanterie ami desd. mineurs nous requerant attandu leur presence dy vouloir bien proceder Lesquels Susnommés Etants aljnstant Comparus nous leur avons fait faire Serment de nous donner Bon Et fidele avis Sur lad. élection Et apres led. Serment fait ont tous d'une commune Et unanime voix dit qu'après avoir murement Examiné Les affaires Concernants les successions des pere Et mere 
desd. mineurs Et les portions quil leur peuvent a Chacun appartenir Suivant la Coutume de paris Suivie En ce pays, Et Eu Egard aux affaires de la maison Et aux dettes passives djcelle montantes a plus de quarante Cinq mille Livres Et les gros frais quil convien devoir faire pour parvenir aud. partage ils ont reconnu que le partage des deux terres qui Composent tout le Bien des deux Successions Seroit tres prejudiciable aux d. mineurs Et aleurs autres freres Et Sœurs Et apres avoir aussy fait Entre Eux tous Lestimation desd. deux terres Et avoir Examiné afond le bien des' parties; ils estiment Et Sont davis pour le plus grand bien Et avantage desd. mineurs de Ceder aud. $S^{r}$ de longue ail fils aisné tous les droits Successifs mobiliers Et jmmobiliers fruits profits Et revenus djceux reseindants Et reseissoires noms Raisons Et actions qui pourroient appartenir aux $d$. mineurs Et toutes les pretentions quils pourroient voir dans les Successions de leurs d. defunts pere et mere En cette Colonie Et ailleurs a l'Exception neanmoins Et Sans aucunem't prejudicier a la portion afferante auSr Etienne Longueuil de maricour preneur dans les dix mille Livres données par mr de Bienville aux Seuls Enfans mâles dud. feu $\mathrm{S}^{r}$ Longueuil; a la Charge par le dit Sieur de longueuil dacquitter lesd. mineurs de toutes les dettes desd. Successions sans aucunes Excepter, Et outre de Conceder achacun desd. mineurs six arpents De front sur trente de profondeur atitre darriere fief relevant de la Baronnie de longueuil, a Choisir Et prendre dans l'Endroit ou Elles jugeront apropos, Et En suivants Les uns les autres dans les terres non Concédées seulement de lad. Baronnie de longueuil Et De leur payer a chacun la Somme de dix mille Livres Scavoir trois mille trois cens trente trois Livres Six Sols huit Deniers afure Et mesure que Chacun d'Eux aura atteint Lage de majorité Et Ensuite continuer dannée En année avec les Intérets de lad. Somme par Chacun an a Raison du denier vingt Suivant Lordre Et Convention Expresse que dans le Cas ou La monoye de cette Colonie pourroit avoir quelque discredit, il Sera loisible aux d. $S^{\text {rs }}$ \& Delles de longueuil de ne point recevoir leurs deniers En avertissant cependant trois mois d'avance, Bien entendu qu'il sera tenu deles payer L'année en Suivante sans cepend ${ }^{t}$ pouvoir Etre Contraint au payement subsequent qui Sera prorogé pour Lannée Suivante Comme aussy que La portion dud. $\mathrm{S}^{\mathbf{r}}$ de longueuil de maricour dans les dix mille Livres provenant de la donation dud. $\mathbf{S}^{\mathbf{r}}$ de Bienville Luy Sera aussy payé par Egale portion Et de la maniere cy dessus Expliquée a Leffet dequoy Et pour En passer contract aud. $\mathrm{S}^{\mathrm{r}}$ de longueuil dans le Cas oujl vouderoit accepter Lesd. propositions jls nomment pour tuteur aux d.mineurs le d. $S^{r}$ de villemonde Et par le d. $S^{r}$ de longueuil a Ete dit qu'Encor qu'il soit Lézé par lesd. propositions Et que Ses frere et Sœurs En recoivent un grand avantage neammoins considerant combien il est important de prendre Les voyes de la douceur il accepte volontier les d. propositions, Et ont 
Signé.

Longueuil

Beaujeu de villemonde

Duquesne

Lantagnac

D’artigny

noyan

Senneville

Devarenne

LaValtrie Tremont

Sur quoy oui le procureur du Roy Eu Ses conclusions Et Suivant jcelles avons omologué Et omologuons Lavis cydessus Et Conformément ajceluy disons quele $\mathrm{dS}^{\mathrm{r}}$ Beaujeu de villemonde est Et demeurera tuteur au d. mineurs Et Successions de leursd. defunts pere Et mere relativement aud. avis Lequel $S^{r}$ de Beaujeu de villemonde pour ce present a volontairement accepte Lad. charge Et fait Le Serment de SEn bien Et fidèlement acquitter Et a Signe. fait les jour Et an susd.

foucher Beaujeu de villemonde

\section{Guiton Monrepos}

Danré De Blanzy

(Registre des tutelles, 1754-55. Archives Judiciaires de Montréal.)

Communication de

Jean-Jacques LEFEBVRE 